\title{
INFLUENCE OF TEXTURE ON POWDER DIFFRACTION
}

\author{
H. J. BUNGE \\ Department of Physical Metallurgy, Technical University of Clausthal, \\ Grosser Bruch 23, D-38078 Clausthal-Zellerfeld, Germany
}

(Received 14 January 1997)

\begin{abstract}
Classical Powder Diffraction usually assumes samples with completely random orientation distribution of the crystallites. It can be generalized to non-random orientation distribution (texture) by introducing a texture factor which enters the expression of the integral intensity directly and serves as a weight function in expressions of peak shift or peak broadening. Hence, all methods of powder diffraction, for instance, phase analysis, crystal structure analysis, stress analysis, particles size analysis, can also be carried out with textured samples.

Textured polycrystalline samples may be considered as being intermediate between single crystals and random powder samples. Hence, they contain information about the directions of reciprocal lattice vectors $r_{(h k l)}^{*}$ which are "averaged out" in random polycrystals, the measured intensities of which depend only on the absolute values $\left|r_{(h k l)}^{*}\right|$. This information can be used, for instance, to separate overlapped structure factors $\left|F_{(h k l)}\right|^{2}$ or to index powder diffraction diagrams.
\end{abstract}

KEY WORDS: Powder diffraction, Texture, Phase analysis, Crystal structure analysis, Stress analysis

\section{INTRODUCTION - DIFFRACTION FROM POLYCRYSTALS}

Polycrystal diffraction means diffraction from samples containing a large number (infinitely many) of small crystallites which cannot be individually distinguished. This applies particularly to two different cases:

- In classical powder diffraction the sample has been prepared from a true (loose) powder. In this case the aggregate parameters of the crystallites in the sample are uninteresting. Ideally, they are chosen to be "as random as possible" both with respect to crystal orientation as well as to spacial arrangement (see e.g. Jenkins and Snyder, 1996).

- In materials science, compact polycrystalline samples must be studied. In this case the aggregate parameters are given. Often they are far from being random and in many cases they have a particular interest for materials characterization (see e.g. Hasek, 1993). This applies particularly to the texture as one of the most important aggregate parameters.

Hence, polycrystal diffraction must take both these cases into account.

In polycrystal diffraction methods essentially three different parameters can be measured experimentally (see e.g. Bish and Post, 1989; Jenkins and Snyder, 1996) as is illustrated schematically in Fig. 1: 


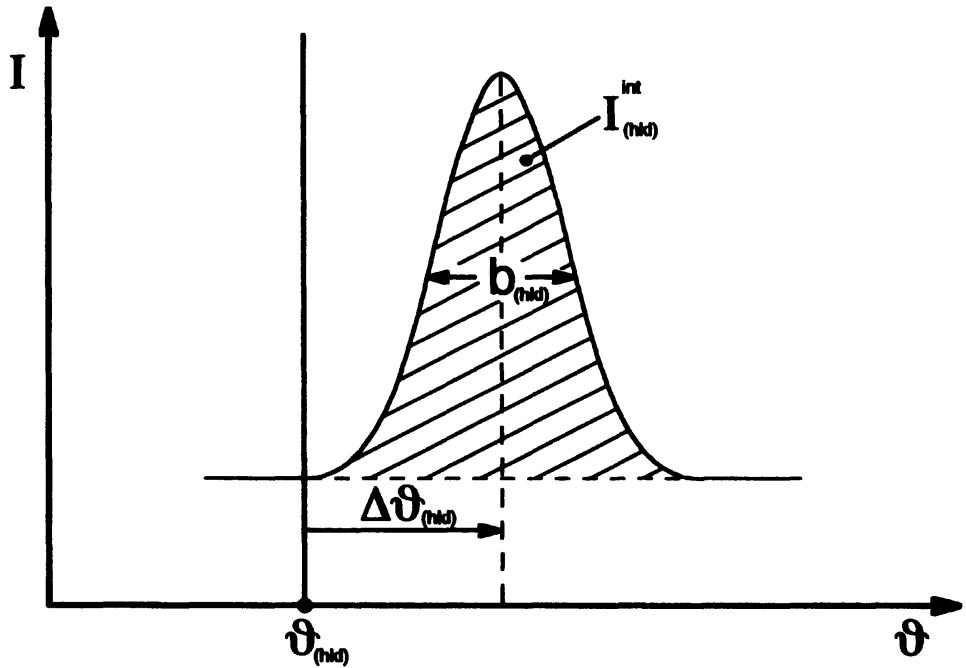

Figure 1 The three main quantities obtained by powder diffraction: Integral intensity, peak shift, peak broadening.

(1) Integral intensity - depending on:

- volume fraction of phases

- crystal structure (structure factors)

- lattice vibrations (temperature factors)

- composition in solid solutions

- ordering in solid solutions

- orientation distribution (texture) texture factor

- lattice defects

- aggregate structure (microabsorption)

(2) Peak position - depending on:

- composition in solid solutions

- thermal expansion

- internal stresses (I kind)

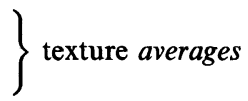

(3) Peak shape (broadening) - depending on:

- internal stresses (II, III kind)

- particle size

- dislocation substructure

- stacking faults<smiles>CCC(C)CC(C)C</smiles>

Measuring any one of these quantities always raises the problem of separating all the influences contributing to that quantity. 
Preferred orientation (texture) has a direct influence only on integral intensity. It may have, however, an indirect influence on the other two quantities. Hence, it must be taken into consideration (as a weight function) when analyzing peak position and peak shape for the parameters on which they depend.

\section{THE RECIPROCAL SPACE OF SINGLE AND POLYCRYSTALS}

The intensity reflected from an ideal single crystal can be expressed by the square of the structure factor $\left|F_{(h k l)}\right|^{2}$ plotted at the reciprocal lattice points $r_{(h k l)}^{*}$ (see e.g. Klug and Alexander, 1962). In a polycrystalline sample, the reciprocal lattices of very many crystals in the orientations $g$ are superposed. Hence, the density distribution (of structure factors) in the reciprocal space of such a sample can be written

$$
\left|\vec{F}\left(r_{(h k l)}\right)\right|^{2}=\oint\left|F\left(g \cdot r_{(h k l)}^{*}\right)\right|^{2} f(g) \mathrm{d} g, \quad r_{(h k l)}=g \cdot r_{(h k l)}^{*},
$$

where $f(g)$ is the crystallite Orientation Distribution Function (ODF). This density distribution consists of a set of spheres with the radii $\left|r_{(h k l)}^{*}\right|$, the pole spheres, with continuous but generally not constant density distribution on these spheres. In the case of random crystallite ODF the density becomes constant on each of the spheres and thus it depends only on $\left|r_{(h k l)}\right|$.

In the most general case, the reciprocal space of a polycrystalline sample is truely three-dimensional. It is the convolution of the reciprocal space of the single crystal with the ODF of the crystallites as expressed in Eq. (1). This is illustrated in Fig. 2 (Bunge, 1994).

\section{Texture Correction}

Many of the classical powder diffraction methods have been worked out on the basis of random crystal ODF (see e.g. Bish and Post, 1989; Jenkins and Snyder, 1996). In
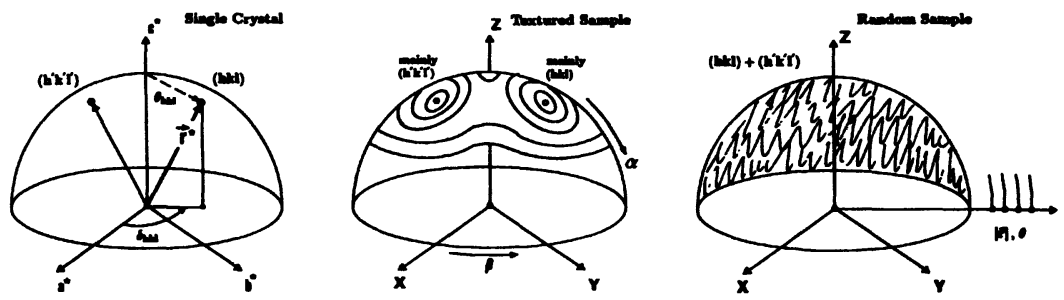

Figure 2 Intensity distribution in the reciprocal space: (a) single crystal, reciprocal lattice points $r_{(h k l)}^{*}$ (three-dimensional reciprocal space); (b) polycrystal with texture, continuous distribution on pole spheres depending on the vector $r_{(h k l)}$ (three-dimensional reciprocal space); (c) random polycrystal, the distribution depends only on $\left|r_{(h k l)}\right|$ (one-dimensional reciprocal space). 
practice, this condition can, however, often not exactly be reached. Nevertheless, it may be assumed as an approximation. The sample will then have a weak residual texture which can be taken into account by a texture correction. After that, the evaluation of the experimental data follows the schedule designed for random samples. Texture correction has been considered by several authors, e.g. Järvinen et al. (1970), Valvoda (1986, 1987), Brokmeier (1991) and Bonarski et al. (1991).

\section{Deconvolution of the Reciprocal Space}

If the texture function $f(g)$ is known and the density $\left|\bar{F}\left(r_{(h k l)}\right)\right|^{2}$ of the textured sample has been measured (depending on the vector $r_{(h k l)}$ and not only on $\left|r_{(h k l)}\right|$ ) then Eq. (1) can, in principle, be deconvoluted for $\left|F\left(r_{(h k l)}^{*}\right)\right|^{2}$. Hence, single crystal diffraction data can, in principle, be obtained from polycrystal measurements making active use of preferred crystal orientation, i.e. texture.

Whereas in the first case, texture is rather considered as a "nuisance" which should be avoided, if possible, in the second case a strong texture is an advantage for density deconvolution according to Eq. (1).

\section{Integral Intensity}

We consider the (corrected) integral intensity measured in a polycrystalline, multiphase sample as a function of the sample direction $\{\alpha \beta\}$ as is illustrated in Fig. 2(b):

$$
I_{(h k l)}^{i}(\alpha \beta)=I_{0} \cdot V^{i} \cdot \frac{1}{\bar{\mu}^{i}} \cdot R_{(h k l)}^{i} \cdot P_{(h k l)}^{i}(\alpha \beta), \quad y=\frac{r_{(h k l)}}{\left|r_{(h k l)}\right|}=\{\alpha \beta\},
$$

where $i$ indicates the phase, $(h k l)$ the reflecting lattice plane, $y=\{\alpha \beta\}$ is the direction of the sample parallel to the diffraction vector $\vec{S}$ referred to the sample coordinate system $K_{A}$, and $I_{0}$ is the intensity of the incident beam. (It is assumed here that the measured intensity has already been corrected for all angular-dependent instrumental intensity factors.)

Equation (2) contains the following four sample-related intensity factors:

$$
\begin{aligned}
V^{i}=\text { Volume fraction } & \rightarrow \text { Phase analysis, } \\
R_{(h k l)}^{i}=\text { Reflectivity } & \rightarrow \text { Structure analysis } \\
& \rightarrow \text { Lattice vibration } \\
& \rightarrow \text { Solid solution } \\
& \rightarrow \text { Ordering } \\
& \rightarrow \text { Lattice defects, } \\
P_{(h k l)}^{i}=\text { Texture factor } & \rightarrow \text { Texture analysis, } \\
\bar{\mu}^{i}=\text { Absorption factor } & \rightarrow \text { Microstructure analysis. }
\end{aligned}
$$

If we are interested in any one of these quantities, the other three must be quantitatively known. 


\section{THE TEXTURE FACTOR}

The texture of a polycrystalline sample is defined by the volume fraction of crystallites having the orientation $g$ of their crystal coordinate system $K_{B}$ with respect to a sample-fixed coordinate sytem $K_{A}$, Fig. 3:

$$
\frac{\mathrm{d} V / V}{\mathrm{~d} g}=f(g) ; \quad g=\left\{\varphi_{1}, \phi, \varphi_{2}\right\}
$$

where $g$ may be expressed in terms of the Euler angles $\left\{\varphi_{1}, \phi, \varphi_{2}\right\}$ relating the crystal coordinate system $K_{B}$ to the sample coordinate system $K_{A}$, Fig. 4. The function $f(g)$ is the ODF introduced in Eq. (1). It is related to the texture factor introduced in Eq. (2) by the relationship

$$
P_{(h k l)}(\alpha \beta)=\frac{1}{2 \pi} \int_{(h k l) \perp\{\alpha \beta\}} f(g) \mathrm{d} \psi=P\left(\Theta_{(h k l)} \gamma_{(h k l)}, \alpha \beta\right),
$$

where $\{\alpha \beta\}$ describes the sample direction parallel to the diffraction vector $\vec{S}$ and $\psi$ is a rotation about the diffraction vector. The angles $\Theta_{(h k l)} \gamma_{(h k l)}$ describe the direction of the reciprocal lattice vector $r_{(h k l)}^{*}$ relative to the crystal coordinate system $K_{B}$ specified in Fig. 2. These angles can formally be considered as continuous variables if we extend $\left|r_{(h k l)}^{*}\right| \rightarrow \infty$, i.e. if we include very high index $(h k l)$.
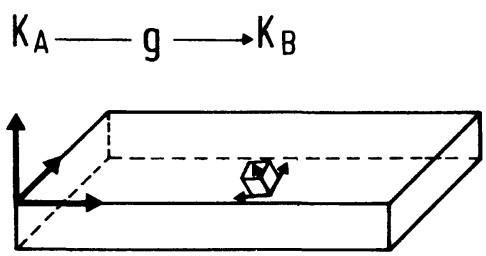

Figure 3 Orientation of a crystallite in a polycrystalline sample with respect to the chosen sample coordinate system $K_{A}$.

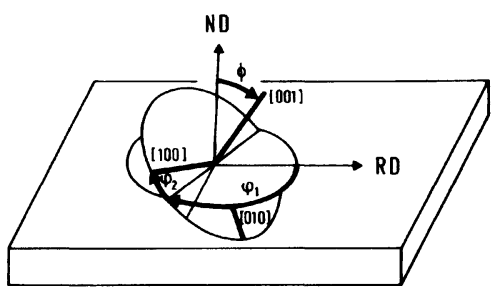

Figure 4 Crystal orientation $g$ can be described by the Euler angles $\varphi_{1}, \phi, \varphi_{2}$ relating two Cartesian coordinate system to each other. Sample coordinate system $K_{A}(R D, T D, N D)$, crystal coordinate system $K_{B}([100][010][001])$. 
Elimination of the Texture Factor by Integration

The simplest way of dealing with the texture factor in Eq. (2) is to eliminate it by integration either over all sample directions $\{\alpha \beta\}$ or over all crystal directions $\left\{\Theta_{(h k l)} \gamma_{(h k l)}\right\}$ using the normalization conditions

$$
\begin{gathered}
\frac{1}{4 \pi} \int_{\alpha}^{\pi} \int_{\beta}^{2 \pi} P_{(h k l)}(\alpha \beta) \mathrm{d} \Omega=1, \text { pole figure, } \\
\frac{1}{4 \pi} \int_{\Theta}^{\pi} \int_{\gamma}^{2 \pi} P(\Theta \gamma)_{\alpha \beta} \mathrm{d} \Omega=1, \quad \text { inverse pole figure. }
\end{gathered}
$$

In principle, the first integral over the "pole figure" $\{\alpha \beta\}$ can be obtained really as an integral, whereas the second one over the "inverse pole figure" $\left\{\Theta_{(h k l)} \gamma_{(h k l)}\right\}$ can only be approximated by the available $(h k l)$.

Applying the integral equation (5) to the (corrected) intensities $I$ in Eq. (2) eliminates the texture factor, and the following evaluation of the data can be carried out as with random samples. Integration according to Eq. (6) over the inverse pole figure must take the $(h k l)$-dependence of the reflectivity $R_{(h k l)}^{i}$ into account (i.e. only $I / R$ can be "integrated" over the inverse pole figure).

\section{Determination of the Texture Factor by Measurements}

If $f(g)$ is known in Eq. (4) the texture factor can be calculated for any sample direction $\{\alpha \beta\}$ and for any $(h k l)$. On the other hand, if the texture factor $P_{(h k l)}(\alpha \beta)$ has been measured for a sufficient number of values $\{\alpha \beta\}$ and/or $(h k l)$ then Eq. (4) can be solved for $f(g)$ in a sufficient approximation. In fortunate cases, this can be achieved with considerably less experimental data than are needed in order to obtain the integrals (5) and (6) with a good accuracy (Bunge et al., 1989; Bunge, 1991).

\section{Texture Representation by Series Expansion}

The texture function $f(g)$ can be expressed in terms of a series expansion using generalized spherical harmonics which are invariant with respect to crystal and sample symmetry (Bunge, 1982).

$$
f(g)=\sum_{\lambda=0(1)}^{L} \sum_{\mu=1}^{M(\lambda)} \sum_{\nu=1}^{N(\nu)} C_{\lambda}^{\mu \nu} \cdot \dot{\bar{T}}_{\lambda}^{\mu \nu}(g) .
$$

Here, the index $\mu$ is related to crystal symmetry and $\nu$ to sample symmetry. We assume that we are not interested in the texture of the sample itself. In this case we can always introduce axial sample symmetry by rapid sample spinning during measurement. Then the index $\nu$ takes on only one value so that we can drop it 
from Eq. (7). Introducing Eq. (7) (with this assumption) into Eq. (4) gives

$$
P_{(h k l)}(\alpha)=\sum_{\lambda=0(2)}^{L} \sum_{\mu=1}^{M(\lambda)} \frac{4 \pi}{2 \lambda+1} C_{\lambda}^{\mu} \cdot \dot{K}_{\lambda}^{\mu}\left(\Theta_{(h k l)} \gamma_{(h k l)}\right) \cdot K_{\lambda}(\alpha),
$$

where $\dot{K}_{\lambda}^{\mu}(\Theta \gamma)$ and $K_{\lambda}(\alpha)$ are spherical harmonics of crystal symmetry and axial sample symmetry, respectively. The index $\lambda$ runs only over even values. The texture (of phase $i$ ) is completely determined by a set of texture coefficients $C_{\lambda}^{\mu}(0 \leq \lambda \leq L)$. These can be calculated from a "sufficient" number of intensity measurements of the reflexions $(h k l)_{m}$ at the sample orientations $\alpha_{n}$ using a least-squares condition

$$
\sum_{(h k l)_{m}} \sum_{\alpha_{n}}\left[I_{(h k l)}(\alpha)-N_{(h k l)} \sum_{\lambda, \mu} \frac{4 \pi}{2 \lambda+1} C_{\lambda}^{\mu} \cdot \dot{K}_{\lambda}^{\mu}(h k l) \cdot K_{\lambda}(\alpha)\right]_{i}^{2}=\min \left[N_{(h k l)}, C_{\lambda}^{\mu}\right]_{i},
$$

where the minimum must be obtained with respect to $N_{(h k l)}$ and $C_{\lambda}^{\mu}$. Here, it is assumed that no overlapping of reflexions $(h k l)$ of all phases $i$ occur. (If this is not true, a similar but more complex expression must be used instead of Eq. (9).)

It may be mentioned here that Eqs. (8) and (9) can also be expressed in terms of all other sample symmetries (i.e. including the index $\nu$ ). See e.g. Bunge $(1977,1982)$. According to Eq. (2) the normalization factor $N_{(h k l)}^{i}$ has the form

$$
N_{(h k l)}^{i}=I_{0} \cdot V^{i} \cdot \frac{1}{\bar{\mu}^{i}} \cdot R_{(h k l)}^{i}=I_{(h k l)}^{i}(\text { random }),
$$

which is identical with Eq. (2) for random samples. Hence, determining the normalization factors $N_{(h k l)}^{i}$ from Eq. (9) reduces powder diffraction of textured samples to the case of random samples.

The solution of Eq. (9) for the unknowns $N_{(h k l)}, C_{\lambda}^{\mu}$ may be considered for two different situations, i.e. full texture analysis and minimum-effort texture analysis.

\section{Full texture analysis}

If the intensities $I_{(h k l)}(\alpha)$ can be measured as (virtually) continuous functions of the angle $\alpha$ then the routine methods of texture analysis (ODF-analysis) can be applied. In this case a solution procedure for Eq. (9) is applied which is based on integration over the measured intensity functions $I_{(h k l)}(\alpha)$ (see e.g. Bunge, 1982). The normalization factors $N_{(h k l)}$ are obtained, in this method, in a direct way based on the normalization condition (5). This requires complete pole figures, i.e. intensities measured in the full range $0 \leq \alpha \leq 90^{\circ}$.

If the intensities $I_{(h k l)}(\alpha)$ are known only in a restricted range $0 \leq \alpha \leq \alpha_{\max }<90^{\circ}$ (incomplete pole figures) then the normalization condition (5) cannot be used explicitly. Nevertheless, a modified procedure can still be used. This procedure starts with an approximation assumption for the non-measured range $\alpha_{\max } \leq \alpha \leq 90^{\circ}$ and 
then repeats the procedure for complete pole figures iteratively. If the measured range $0 \leq \alpha \leq \alpha_{\max }$ is large enough then this iterative procedure converges quite well (see e.g. Dahms and Bunge, 1989).

Routine computer programs for "full texture analysis" are available depending on crystal and sample symmetry (see e.g. Dahlem-Klein et al. (1993) for cubic crystal symmetry). The axial sample symmetry can easily be introduced as a special case (subsymmetry) of the (most frequently used) orthorhombic sample symmetry.

"Full texture analysis" is based on the measurement of "pole figures" as continuous functions of the sample angles $\alpha, \beta$ (in the particular case of axial sample symmetry only the angle $\alpha$ ). It usually requires only a low number of reflexions $(h k l)$ of which pole figures must be measured. The total number of measured intensity values $I_{(h k l)}(\alpha)$ included in the solution of Eq. (9) is usually much higher than the number of unknown quantities, i.e. $C_{\lambda}^{\mu}$ and $N_{(h k l)}$. Full texture analysis thus uses a good deal of overdetermination (redundance) for the determination of the unknowns $C_{\lambda}^{\mu}$ and $N_{(h k l)}$.

In full texture analysis the degree $L$ of series expansion can be chosen. Often the value $L$ is chosen "high enough" so as to be "on the safe side" for an unknown degree of texture sharpness. Hence, full texture analysis requires a texture goniometer which allows the measurement of intensities as a continuous function of the angles $\alpha, \beta$ (or at least the angle $\alpha$ ).

\section{"Minimum-effort" texture analysis}

In many cases it is, however, desirable to obtain the texture coefficients $C_{\lambda}^{\mu}$ in Eq. (9) from the lowest possible number of intensity measurements $I_{(h k l)}(\alpha)$, particularly only from a few (isolated) angular values $\alpha_{n}$ as was assumed in Eq. (9). This situation is particularly encountered in routine phase analysis from true powders. In this case it may be assumed that it is possible to prepare a sample with a very flat texture which can be sufficiently characterized by a low number of coefficients $C_{\lambda}^{\mu}$ in Eq. (9). In this case another solution procedure of Eq. (9) can be used which is not based on integration over the angle a (see e.g. Bunge, 1977; 1990; Bunge and Wang, 1987). Also in this procedure some redundance of measured intensity values with respect to the unknowns $C_{\lambda}^{\mu}$ and $N_{(h k l)}$ is required. However, the degree of redundance is to be kept as low as possible (with respect to the required accuracy of the values $C_{\lambda}^{\mu}$ and $\left.N_{(h k l)}\right)$.

The number of unknowns, and hence the number of required intensity values, can be reduced by:

(1) using the flattest possible texture in order to have the lowest number of unknown texture coefficients $C_{\lambda}^{\mu}$ (i.e. the lowest value $L$ );

(2) taking the $(h k l)$-dependence of the normalization factors $N_{(h k l)}^{i}$ into account, such that only one normalization factor $N^{i}$ (for each phase $i$ ) remains unknown in Eq. (9).

In order to fulfill condition (2) we put

$$
R_{(h k l)}^{i}=R^{i} \cdot r_{(h k l)}^{i} \quad\left(\text { with } r_{(\max )}^{i}=1\right)
$$


where $r_{(h k l)}^{i}$ are relative intensities (of the phase $i$ ) and $R^{i}$ are reference intensities of the phases $i$ (referred to a standard phase, e.g. $\mathrm{Al}_{2} \mathrm{O}_{3}$ ). If the $r_{(h k l)}^{i}$ are known the intensities $I_{(h k l)}(\alpha)$ can be divided by them so that only one normalization factor $N^{i}$ remains in Eq. (9). (The quantities $r$ in Eq. (11) are different from the reciprocal lattice vectors $r$ of Eq. (1).)

The knowledge of $R^{i}$ does not further simplify Eq. (9) since the normalization factor $N^{i}$ still contains other unknown parameters, particularly $V^{i}$ (as well as $\bar{\mu}^{i}$ depending on $V^{i}$ ). Hence, this factor can no more be dropped from Eq. (9). The knowledge of $R^{i}$ is, however, needed in order to solve Eq. (10) for the volume fractions $V^{i}$ in phase analysis.

\section{Texture Degree and Convergence Value $L$}

The number of texture coefficients $C_{\lambda}^{\mu}$ in Eqs. (8) and (9) depends on the required degree $L$ (at which convergence of the series has been reached within a required accuracy) and on the quantity $M(\lambda)$ which depends on crystal symmetry as is shown in Fig. 5. The total number of coefficients $C_{\lambda}^{\mu}$ up to $\lambda=L$ is then obtained as given in Table 1. Weak residual textures, as they may be obtained by trying to prepare random samples, can often be described by the lowest $L$-value (e.g. 2 or 4 , respectively). In materials science, however, convergence values of $L=22$ (and higher) are frequently necessary.

\section{Texture Model Functions}

Sometimes it is possible to represent the texture function by a function of a specific type which then depends only on a few parameters (which may be less than the

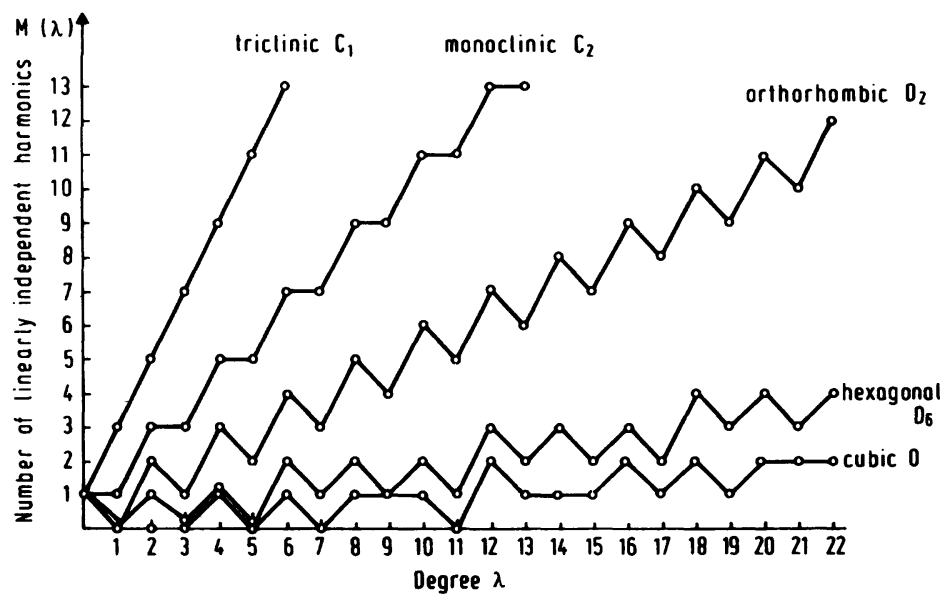

Figure 5 The quantity $M(\lambda)$ as a function of $\lambda$ for different crystal symmetries. 
Table 1 The total number of texture coefficients $C_{\lambda}^{\mu}$ (for axial symmetry) as a function of the expansion degree $L$ and for different crystal symmetries (for $L \rightarrow \infty$ also the number of coefficients goes to $\rightarrow \infty$ )

\begin{tabular}{llccc}
\hline & & \multicolumn{3}{c}{ Crystal Symmetry } \\
\cline { 3 - 5 } & & Cubic & Orthorhombic & Triclinic \\
\hline Random Sample & $L=0$ & 0 & 0 & 0 \\
Residual Texture & $L=2$ & 0 & 2 & 5 \\
(prepared, to be & $L=4$ & 1 & 5 & 14 \\
random) & $L=6$ & 2 & 9 & 27 \\
Strong Texture & $L=22$ & 15 & 77 & 275 \\
\hline
\end{tabular}

number of coefficients $C_{\lambda}^{\mu}$ in Eq. (9)). For example, the texture may be characterized by one preferred orientation $g_{0}=\left\{\varphi_{1}^{0} \phi^{0} \varphi_{2}^{0}\right\}$ with some spread with the spread parameter $\omega$ about it. Particularly simple is a Gaussian spread. Then the texture function has the form

$$
f_{g_{0} \omega}(g)=f_{\max } \cdot \mathrm{e}^{-(|\Delta g| / \omega)^{2}}, \quad g=\Delta g \cdot g_{0} .
$$

In the case of axially symmetric textures the angle $\varphi_{1}^{0}$ is not defined. Hence, the model texture Eq. (12) contains three free parameters $\phi^{0} \varphi_{2}^{0} \omega$. Furthermore, it can often be assumed that the preferred orientation, i.e. $\phi^{0} \varphi_{2}^{0}$ is known. Then we are left with only one free parameter $\omega$. (The value $f_{\max }$ follows from the normalization condition of $f(g)$.)

It must, however, be mentioned that besides the Gaussian spread function many other functions such as the well-known March function (March, 1932) have been suggested in the literature (see e.g. Dollase, 1986), and that the accuracy of the results depends on the excact knowledge of the spread function.

\section{DETERMINATION OF THE REFLECTIVITIES}

The reflectivities $R_{(h k l)}^{i}$, as defined in Eq. (2), may depend on a number of parameters which may not be totally known as was mentioned already in the context of Eq. (2). If we assume that the parameters of the ideal crystal lattice are known, i.e. crystal structure, lattice vibration (temperature), solid solution and ordering, then still lattice defects depending on the actual state of the material (due to mechanical and/ or thermal treatment of the sample) may have an unknown influence. Reflectivities may be obtained in the following ways:

- ab-initio calculation of the reflectivities from structure data (lattice defects not included),

- reflectivities taken from reference file (JCPDS) (lattice defects as in the reference sample),

- experimental determination of the actual reflectivities (lattice defects as in the actual sample). 
Particularly for this latter methods many variants using internal or external standards are known. Full texture analysis as described above can contribute to this problem. As it was mentioned, full texture analysis can be carried out without knowing the normalization factors $N_{(h k l)}^{i}$ in Eq. (9). Rather these factors are obtained as a result of this procedure. The reflectivities can then be obtained from Eq. (10) with $V^{i}=1$. The relative intensities $r_{(h k l)}^{i}$ in Eq. (11) can even be obtained from texture measurements of phase mixtures with $V^{i} \neq 1$.

\section{INFLUENCE OF SAMPLE AGGREGATE PARAMETERS}

Equation (2) is valid under some idealizing assumptions, concerning the polycrystalline aggregate, which may be very restrictive, indeed. Some of the most important aggregate parameters will be considered in the following.

\section{Particle statistics}

The volume fractions $V^{i}$ are (at the best) the fractions of the phases $i$ within the irradiated volume of the sample. If the phase particles are not very small, these volume fractions are subject to statistical fluctuations which can be expressed in terms of the number of particles $n^{i}$ in the irradiated volume. Hence, the uncertainty of the volume fractions may be expressed by

$$
\frac{\mathrm{d} V^{i}}{V^{i}}=\frac{\mathrm{d} n^{i}}{n^{i}}=\left[n^{i}\right]^{-1 / 2} \quad\left(n^{i}=\text { Number of irradiated particles }\right) .
$$

\section{Crystallite statistics}

Similar statistical fluctuations may apply to the texture factor $P$ depending on the number of crystallites, the size of which may be identical with the size of particles (single crystal particles) or may be smaller. The uncertainty of the texture factor is thus of the order

$$
\frac{\mathrm{d} P}{P}=\frac{\mathrm{d} n}{n}=[n]^{-1 / 2} \quad(n=\text { number of reflecting crystallites }) .
$$

It must be mentioned that $n$ in Eq. (14) means the number of crystallites in reflecting orientation the volume of which may be smaller (by several orders of magnitude) than the (already small) irradiated volume. In this case the texture factor $P_{(h k l)}(\alpha)$ is not a smoth function of $\alpha$, rather it may be a "ragged", "peaky" function.

\section{Particle size}

Independent of particle statistics, also particle size may have a direct influence. In Eq. (2), $\bar{\mu}^{i}$ is the mean absorption factor for beams reflected in the phase $i$. For this mean value, two limiting cases can be considered, i.e. for particles which are big or 
small compared to the penetration depth of the used radiation, as is illustrated in Fig. 6. The following expressions are obtained in these two cases:

$$
\bar{\mu}^{i}= \begin{cases}\bar{\mu}_{\mathrm{big}}^{i}=\mu^{i} & \text { (big particles), } \\ \bar{\mu}_{\mathrm{small}}^{i}=\bar{\mu}=\sum \mu^{j} \cdot V^{j} & \text { (small particles). }\end{cases}
$$

Figure 7 shows, for instance, the situation for a mixture of $\mathrm{CaCO}_{3}$ and $\mathrm{SiO}_{2}$ particles. Unfortunately, real particle sizes are often between these two limiting cases. In this intermediate range exact expressions for $\bar{\mu}^{i}$ are not known. Also, such expressions (if they were known) would depend on many parameters, the exact values of which for the actual sample are unknown (see e.g. Reefman, 1997).

\section{Particle shape}

If the particles deviate from equiaxial shape, they may be "big" in one direction and "small" in another one. In this case $\bar{\mu}^{i}$ may assume values between the limiting values of Eq. (15) depending on $\alpha \beta$ and particularly also on a rotation $\psi$ about the diffraction vector $\vec{S}$ as shown in Fig. 8. In this case also $I_{(h k l)}^{i}$ in Eq. (2) becomes $\psi$ dependent (which is, however, not considered in Eq. (2)). The effect of anisotropic absorption due to anisometric particle size is extremely strong in the case of lamellar eutectic alloys, Fig. 9. It must, however, be assumed to be a quite general effect since powder particles are often plate- or needle-like.
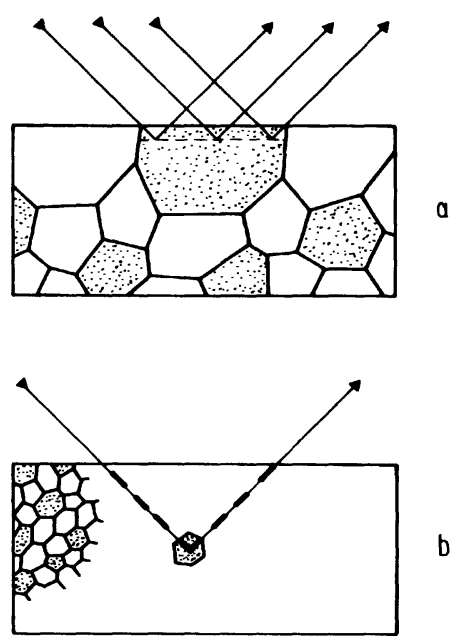

Figure 6 Absorption of X-rays reflected in one phase of a two-phase mixture: (a) big particles, (b) small particles. 


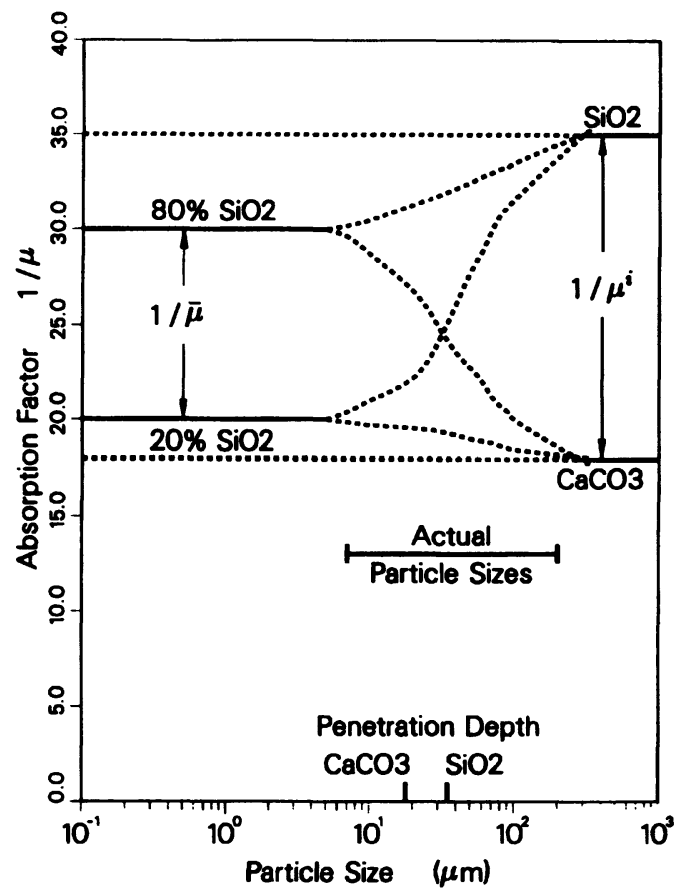

Figure 7 The absorption factor $1 / \bar{\mu}^{i}$ for different volume fractions of $\mathrm{SiO}_{2}$ and $\mathrm{CaCO}_{3}$ as a function of particle size. The penetration depth of $\mathrm{CuK}_{\alpha}$ radiation in both materials is also indicated.

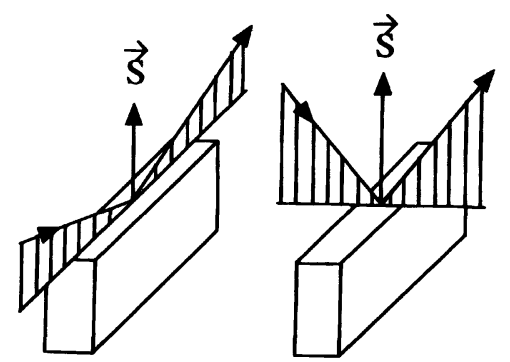

Figure 8 Influence of particle shape on the absorption factor. Diffraction plane parallel to the: (a) long axis of the particle (big-particle case), (b) short axis of the particle (small-particle case). 

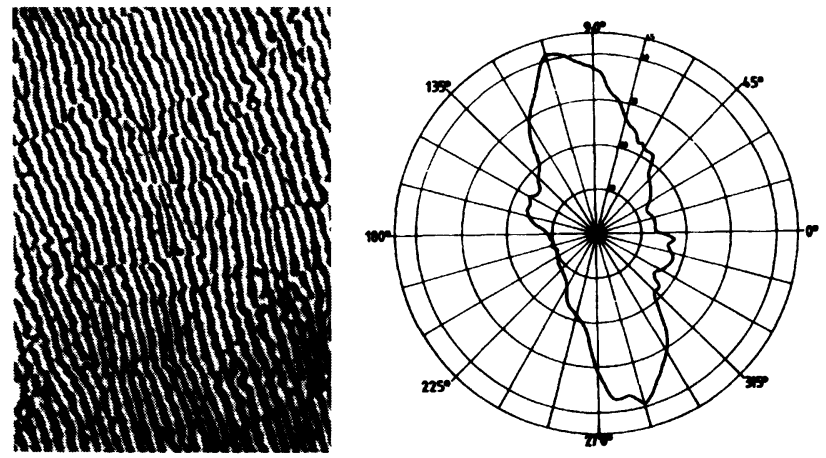

Figure 9 The absorption factor $1 / \bar{\mu}^{i}$ in a lamellar $\mathrm{Pb}-\mathrm{Sn}$ alloy as a function of the rotation $\psi$ about the diffraction vector (as illustrated in Fig. 8).

\section{Particle arrangement}

A similar effect as that caused by anisometric particle shape may also occur by anisotropic particle arrangement. For example spherical particles aligned in strings may also lead to $\bar{\mu}^{i}$ depending on $\{\alpha \beta \psi\}$.

\section{Surface inhomogeneity}

Samples may show surface inhomogeneities with respect to the mentioned aggregate parameters, e.g. particle size, volume fraction, and particularly particle orientation. Since often these inhomogeneities are in the order of magnitude of the X-ray penetration depth this leads to $\alpha$-dependent averages of all these quantities when measuring at varying sample tilt angles (see e.g. Fiala, 1993). For the treatment of textures, this would mean that Eq. (8) (considered as a function of $\alpha$ ) is no longer correct. Rather, also the coefficients $C_{\lambda}^{\mu}$ in this equation would then be $\alpha$-dependent. It may be mentioned that particular measuring techniques have been developed in which the "information" depth becomes independent of the angle $\alpha$ in order to avoid this effect (see e.g. Bonarski et al., 1994).

\section{Influence of Non-texture Effects on Texture Determination}

The effects of crystallite statistics, particle shape, particle arrangement, and texture inhomogeneity introduce intensity factors depending on $\{\alpha \beta \psi\}$ which are not contained in Eq. (2). Particularly, they may introduce $\alpha$-dependent errors of the intensity $I_{(h k l)}(\alpha)$ in Eq. (9) which the minimization procedure in Eq. (9) tries to interprete in terms of a (non-real) texture. This effect is particularly serious in the case of "minimum effort" texture analysis where there is not enough redundance to eliminate it. In this case the results may even become worse than without taking a texture factor into account. In the case of "full texture analysis" there is sufficient 
redundance such that the minimization procedure can recognize intensity variations due to texture and can eliminate others, the $\{\alpha \beta \psi\}$-dependence of which cannot be interpreted in terms of any (hypothetical) texture.

\section{Neutron Diffraction}

It is important to mention that all sample aggregate parameters which may falsify the results of powder diffraction decrease with increasing penetration depth of the used radiation. In most practical cases they are virtually absent when the penetration depth reaches the $\mathrm{cm}$ range. This is the case for neutron diffraction. Hence, highest precision in all powder diffraction methods can be reached by using neutron diffraction. Of course, then sample volumes in the $\mathrm{cm}^{3}$ range must be available (see e.g. Bunge, 1989).

\section{TEXTURES OBSERVED IN POWDER SAMPLES}

In order to estimate the necessary number of intensity measurements in a "minimum effort" texture analysis the texture degree obtained by preparing samples from given powders was investigated (Gao et al., 1992; 1993). Powders of different materials were pressed into a sample holder to make a flat sample. Thereby different techniques were used but without taking precautions to avoid preferred orientation. The results are shown in Table 2. This table gives the maximum value of the texture factor together with an estimated convergence value $\lambda_{\text {conv }}$ necessary for the representation of such texture. The weakest texture was observed in Al-powder but, nevertheless, even in this case the maximum value of the texture factor was 1.3 which means an error of $30 \%$ in the measured intensity values if the texture factor is not taken into account.

Parameters influencing the texture in a compacted powder sample were particularly:

- particle size and shape and their distribution functions,

- mechanical properties of the particles (plastic deformation, cleavage),

Table 2 Maximum value of the texture factor and estimated convergence value $\lambda_{\text {conv }}$ for powder samples of different materials

\begin{tabular}{llclcc}
\hline Material & Symmetry & $\begin{array}{c}\text { Particle Size } \\
{[\mu \mathrm{m}]}\end{array}$ & Particle Shape & $\begin{array}{c}\text { Maximum } \\
\text { Pole Density }\end{array}$ & $\begin{array}{c}\text { Series Conver- } \\
\text { gence } \lambda_{\text {conv }}\end{array}$ \\
\hline $\mathrm{MoS}_{2}$ & Hexagonal & - & Plate & 5.2 & 8 \\
$\mathrm{ZnO}_{\mathrm{Fe}_{3} \mathrm{O}_{4}}$ & Hexagonal & 43.5 & Globular & 1.9 & 4 \\
$\mathrm{SnO}$ & Cubic & 47.5 & Globular & 3.6 & 6 \\
$\mathrm{Graphite}$ & Tetragonal & - & - & 1.6 & 4 \\
$\mathrm{Cr}_{2} \mathrm{O}_{3}$ & Rhombonal & - & Plate & 7.2 & 12 \\
$\mathrm{Cu}_{2} \mathrm{O}$ & Cubic & 44.5 & Globular & 2.4 & 2 \\
$\mathrm{Gypsum}$ & Monoclinic & 47.5 & Globular & 2.4 & 4 \\
$\mathrm{Al}_{2} \mathrm{O}_{3}$ & Trigonal & - & Globular & 4.1 & 6 \\
Aspirine & - & - & - & 6.3 & 8 \\
$\mathrm{Al}$ & Cubic & 39.0 & Irregular & 10.9 & 14 \\
\hline
\end{tabular}




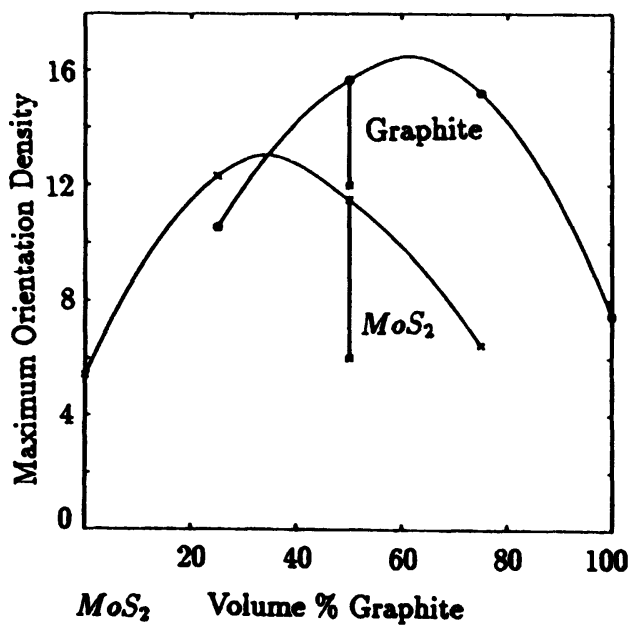

Figure 10 The maximum orientation density in mixtures of graphite and molybdenum sulfide as a function of volume fraction with equal preparation technique. The deviating points correspond to a threephase mixture containing $\mathrm{Al}$ as the third phase.

- embedding medium (air, liquid, binder phase),

- mode of pressing (e.g. uniaxial pressure, shear, front or rear side),

- volume fractions $V^{i}$ in mixtures (interaction of particles of different phases).

This latter effect was particularly strong in mixtures of graphite and molybdenum sulfide as illustrated in Fig. 10 but it was also observed in many other mixtures. It was also found that "nominally" identical powders and "nominally" identical preparation techniques gave significantly different values of the texture factors.

In some cases, textures consisting of more than one preferred orientation were found as is seen for $\mathrm{MoS}_{2}$ in Fig. 11. In this case the method of "single peak model functions", as described above, cannot be used.

Finally, the observed textures often were inhomogeneous particularly near to the sample surface.

\section{DETERMINATION OF INTEGRATED INTENSITIES}

In Eq. (2) integrated (corrected) intensities $I_{(h k l)}^{i}(\alpha \beta)$ were assumed. They are defined with respect to the intensity taken as a function of the Bragg angle $\vartheta$ which can be written, in analogy to Eq. (2), in the form

$$
I(\vartheta \alpha \beta)=\sum_{i} \sum_{(h k l)} I_{(h k l)}^{i}(\alpha \beta) \cdot B\left(\vartheta-\vartheta_{(h k l)}^{i}, b(\vartheta \alpha)\right)
$$




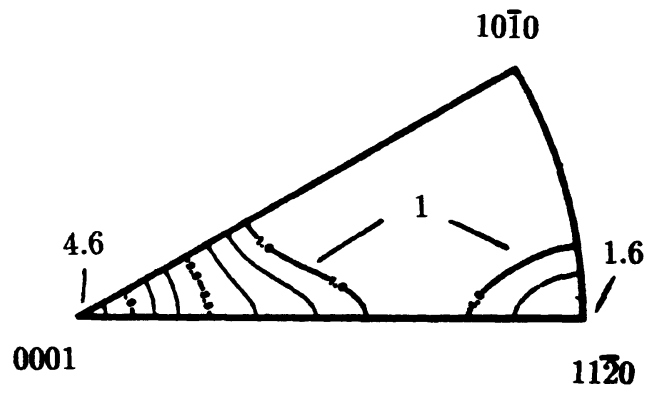

Figure 11 Orientation distribution of $\mathrm{MoS}_{2}$ pressed into a sample holder. The texture shows two preferred orientations.

Thereby $B$ is the normalized peak profile function of the reflex $(h k l)^{i}$ centered at $\vartheta_{(h k l)}^{i}$ and having a peak width $b$ which depends on the Bragg angle $\vartheta$ and very strongly on the sample tilt angle $\alpha$. It is assumed here that the peak profile function is truely instrumental, i.e. it does not depend on the properties of the sample. (This latter assumption excludes the influences described in points (2) and (3) in the introduction.) Hence, the function $B$ is assumed, here, to be known for all $\vartheta$ and $\alpha$.

In order to obtain integral intensities from Eq. (16) three different cases may be considered.

\section{Non-overlapped peaks}

In this case the peak profile of the reflex $(h k l)^{i}$ does not overlap with any other neighboring peak. This is particularly to be proofed for high tilt angles $\alpha$ where strong peak broadening occurs. The integrated intensity is then obtained by integrating Eq. (16) over $\vartheta$ in the vicinity of $\vartheta_{(h k l)}^{i}$ (with $\alpha \beta$ constant):

$$
I_{(h k l)}^{i}(\alpha \beta)=\int_{\vartheta_{(h k l)}-\Delta \vartheta_{\max }}^{\vartheta_{(h k l)}+\Delta \vartheta_{\max }} I(\vartheta \alpha \beta) \mathrm{d} \vartheta, \quad \int B(\vartheta) \mathrm{d} \vartheta=1 .
$$

In this case only the normalization condition of the peak profile function is essential. The particular form of the profile function is not important. In this case "physical" integration can be used, e.g. by using a wide detector entrance slit which accepts the whole peak profile, even the broadened one in the tilted position.

\section{Moderately overlapped peaks}

In this case it is assumed that several peaks $(h k l)^{i}$, neighboring in $\vartheta$, may be overlapped in $\vartheta$ but that their distances $\Delta \vartheta$ are not too small compared with the widths $b(\vartheta \alpha)$ of the peak profile functions, particularly including high tilt angles $\alpha$. In this case peak profile analysis over $\vartheta$ can be carried out using a least squares 
condition over $\vartheta$,

$$
\int_{\vartheta_{\min }}^{\vartheta_{\max }}\left[I^{\operatorname{Exp}}(\vartheta \alpha \beta)-I^{\operatorname{Mod}}(\vartheta \alpha \beta)\right]^{2} \mathrm{~d} \vartheta=\min
$$

with $I^{\text {Mod }}$ according to Eq. (16).

In this case the functions $B$ must be known. The integrated intensities $I_{(h k l)}^{i}(\alpha \beta)$ are then obtained by minimizing Eq. (18) (see e.g. Wcislak and Bunge, 1996) in this case the intensities $I^{\operatorname{Exp}}$ must be measured with the highest possible resolution in $\vartheta$. This method can be used, for instance, in combination with a position sensitive detector.

\section{Strongly overlapped peaks}

In this case the peak positions may be very close to each other or even identical in $\vartheta$. Peak separation is then obtained with the help of the texture, i.e. over the sample angles $\alpha \beta$. For this purpose the intensity $I(\vartheta \alpha \beta)$ is expressed in the form

$$
I(\vartheta \alpha \beta)=\sum_{i} \sum_{(h k l)} I_{(h k l)}^{i}(\text { rand }) \cdot B\left(\vartheta-\vartheta_{(h k l)}^{i}, b(\vartheta \alpha)\right) \cdot P_{(h k l)}^{i}(\alpha \beta) .
$$

Here it is assumed that the textures $f^{i}(g)$ of the phases $i$ are already known. The texture factors $P_{(h k l)}^{i}(\alpha \beta)$ are then given by Eq. (4). The unknown quantities in Eq. (19) are the integral intensities for random orientation distribution defined by Eq. (2) with $P_{(h k l)}^{i}=1$. (It must be mentioned that in Eq. (19) anisotropic absorption has been excluded, i.e. $\bar{\mu}^{i}$ has been assumed to be orientation independent.) The random intensities are then obtained by a least squares condition over all three angles $\vartheta \alpha \beta$

$$
\int_{\vartheta_{\min }}^{\vartheta_{\max }} \int_{\alpha} \int_{\beta}\left[I^{\mathrm{Exp}}(\vartheta \alpha \beta)-I^{\mathrm{Mod}}(\vartheta \alpha \beta)\right]^{2} \mathrm{~d} \vartheta \sin \alpha \mathrm{d} \alpha \mathrm{d} \beta=\min
$$

with $I^{\text {Mod }}$ according to Eq. (19). In this case the texture must be known additionally to the peak profile function. Peak separation is then essentially based on the $\alpha \beta$ dependence of the experimental intensity (see e.g. Hedel et al., 1997). Because of the large number of experimental data needed in this case, the measurements virtually require a position sensitive detector.

\section{PEAK SHAPE AND POSITION}

In Eq. (1) the density in the reciprocal space of a polycrystalline (single phase) sample was expressed in terms of the structure factors of an ideal single crystal and the orientation distribution function of the crystallites. This expression can also be generalized for the case of non-ideal crystals. In this case the density in the reciprocal space in the vicinity of the ideal reciprocal lattice point $r_{(h k l)}^{*}$ can be written in the 
form

$$
\left|\tilde{F}\left(r^{*}\right)\right|_{g}^{2}=\left|F_{(h k l)}\right|^{2} \cdot D_{(h k l)}\left(r^{*}-r_{(h k l)}^{*}\right)_{g}
$$

The "distortion" functions $D_{(h k l)}$ may contain peak shift and peak broadening as is indicated in Fig. 12. They may also contain intensity changes. This means that the integral over the function $D$ is not necessarily equal to one. Strictly speaking, each individual crystallite may have its own particular distortion functions. In polycrystalline samples individual crystallites of the same orientation cannot be distinguished. Hence, it is only meaningfull to define distortion functions for the group of all crystallites having the same orientation $g$ as is indicated in Eq. (21) by the index $g$ (Fig. 12(d)). Then Eq. (1) can be generalized in the form

$$
\left|\bar{F}\left(r_{(h k l)}\right)\right|^{2}=\oint\left|\tilde{F}\left(g \cdot r^{*}\right)\right|_{g}^{2} \cdot f(g) \mathrm{d} g
$$

The distribution in the reciprocal space of the polycrystalline sample thus contains peak shift and peak broadening of the reflex $(\mathrm{hkl})$ both as a function of the direction $\alpha \beta$ of the vector $r_{(h k l)}$. This is shown schematically in Fig. 12(e) (Bunge, 1997). Peak shift and peak broadening can be measured as a function of the angles $\alpha \beta$ (see e.g. Wcislak and Bunge, 1996). The texture may also be assumed to be known from measured distribution functions of the integral intensity Eq. (2). Then the question arises if it is possible to deconvolute Eq. (22) for the distortion function $D$ in Eq. (21). In the most general case where each crystal orientation $g$ may have its own unknown distortion functions, a unique solution cannot be expected. Nevertheless, there are many special cases where it is known that the distortion functions $D$ must have a particular form with only a few free (unknown) parameters. Then it may be possible to determine these parameters from measurements of peak shift and/or peak broadening pole figures along with integral intensity pole figures. Examples of that are the determination of internal stresses of $I$ kind from peak shift measurements and of stresses of II and III kind and/or particle size from peak broadening. It is clear from Fig. 12 that the calculation of stresses and/or particle size from peak shift and/ or broadening pole figures depends essentially on the texture.

\section{Internal Stresses of I Kind}

According to Hooke's Law internal stresses give rise to strains which, in turn, lead to peak shift. Hooke's Law for a single crystal (assuming homogeneous stress and strain in the crystal) may be written in two equivalent forms:

$$
\sigma_{i j}=C_{i j k l} \cdot \varepsilon_{k l}, \quad \varepsilon_{i j}=S_{i j k l} \cdot \sigma_{k l}, \quad\left[S_{i j k l}\right]=\left[C_{i j k l}\right]^{-1} .
$$

Thereby $\sigma$ is the stress tensor, $\varepsilon$ is the strain tensor and $C$ and $S$ are the elasticity tensors (i.e. stiffness and compliance tensor, respectively). The tensors $C$ and $S$ are 
a)

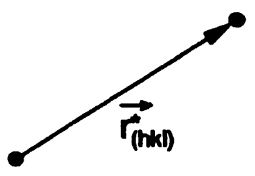

b)

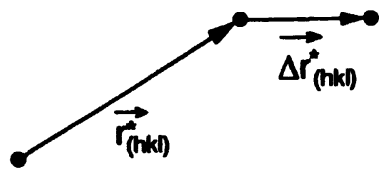

c)

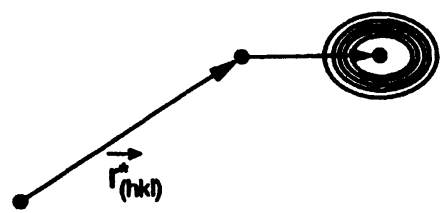

d)

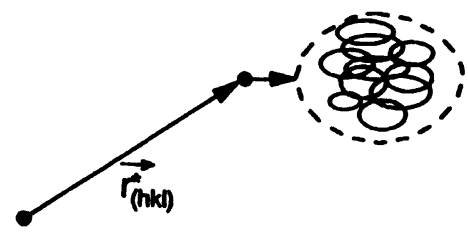

ө)

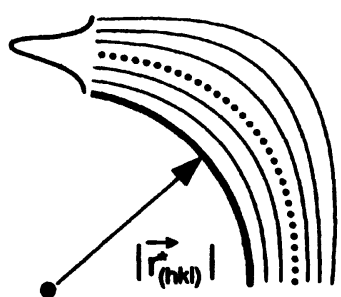

Figure 12 The distribution $D_{(h k l)}$ (distortion function) in the vicinity of the reciprocal lattice point ( $h k l$ ) of a (non-ideal) single crystal and peak shift and broadening in a polycrystalline sample: (a) the ideal reciprocal lattice point $r_{(h k l)}^{*}$; (b) shift of the point $r_{(h k l)}^{*}$ by $\Delta r_{(h k l)}^{*}$; (c) shift and broadening in one crystallite; (d) shift and broadening in a group of crystals with the same orientation $g$; (e) peak shift and broadening in the polycrystalline sample.

inverse to each other. All tensor components are referred to the chosen coordinate system. If we want to average over all crystallites with different crystal orientations we have to choose the (common) sample coordinate system $K_{A}$ for all of them. On the other hand, the elasticity tensors are usually tabulated by components referred to the 
crystal coordinate system $K_{B}$, Fig. 3 . Hence, the tensor transformation law is needed:

$$
\begin{aligned}
& C_{i j k l}(g)=C_{\text {mnop }}^{0} \cdot T_{i j k l m n o p}(g), \\
& S_{i j k l}(g)=S_{\text {mnop }}^{0} \cdot T_{i j k l m n o p}(g),
\end{aligned}
$$

where $T$ is an "orientation tensor" expressed in terms of the transformation matrix $g=\left[g_{i j}\right]$ which relates the crystal coordinate system $K_{B}$ to the sample coordinate system $K_{A}$. It is

$$
T_{i j k l m n o p}(g)=g_{i m} \cdot g_{j n} \cdot g_{k o} \cdot g_{l p}
$$

The components $g_{i j}$ can be expressed in terms of the Euler angles of Eq. (3).

In a polycrystalline material the crystallites interact elastically. Hence stresses as well as strains are not homogeneous as was assumed in Eq. (23). Rather they will depend on crystal orientation $g$ and they may even be inhomogeneous inside a crystal.

Peak shift $\Delta \vartheta_{(h k l)}(\vec{y})$ of the peak $(h k l)$ measured in the sample direction $\vec{y}=\left[y_{i}\right]=\{\alpha \beta\}$ (i.e. the peak shift pole figure) is related to the average strain of the reflecting crystals taken in the direction $\vec{y}$, i.e. the lattice strain pole figure (Hoffmann et al., 1986).

$$
\Delta \vartheta_{(h k l)}(\vec{y})=-\tilde{\varepsilon}_{(h k l)}(\vec{y}) \cdot \tan \vartheta_{(h k l)},
$$

whereby the average strain component in the direction $\vec{y}$ is given by the average strain tensor

$$
\tilde{\varepsilon}_{(h k l)}(\vec{y})=\tilde{\varepsilon}_{i j} \cdot y_{i} \cdot y_{j}
$$

and the average strain tensor $\tilde{\varepsilon}_{i j}$ is given by the integral of the individual strain tensors taken over the reflecting crystals

$$
\tilde{\varepsilon}_{i j}=\int_{\vec{y} \perp(h k l)} \varepsilon_{i j}(g) \mathrm{d} \psi
$$

The orientation dependence of the strain $\varepsilon_{i j}(g)$ is not very well known. In fact it depends on the texture as well as on the local arrangement of crystallites of different orientations $g$ (see e.g. Kiewel et al., 1995). Hence, two limiting hypotheses are often used:

$$
\begin{aligned}
\varepsilon_{i j}(g)=\bar{\varepsilon}_{i j}=\text { const. } & \text { (Voigt assumption), } \\
\sigma_{i j}(g)=\bar{\sigma}_{i j}=\text { const. } & \text { (Reuss assumption). }
\end{aligned}
$$

With these two assumptions the lattice strain pole figure $\tilde{\varepsilon}_{(h k l)}(\vec{y})$ can be expressed in terms of the average (macroscopic) stress tensor $\bar{\sigma}_{i j}$ (see e.g. Brakman, 1987; Barral 
et al., 1987; Bunge, 1988; Matthies, 1996):

$$
\begin{aligned}
& \tilde{\varepsilon}_{(h k l)}(\vec{y})^{\text {Voigt }}=\left[C_{m n o p}^{0} \cdot \bar{T}_{i j k l m n o p}^{\text {Voigt }}\right]^{-1} \cdot y_{i} \cdot y_{j} \cdot \bar{\sigma}_{k l} \quad(\text { Voigt }), \\
& \tilde{\varepsilon}_{(h k l)}(\vec{y})^{\text {Reuss }}=S_{m n o p}^{0} \cdot \bar{T}_{i j k l m n o p}^{\text {Reuss }} \cdot y_{i} \cdot y_{j} \cdot \bar{\sigma}_{k l} \quad(\text { Reuss }),
\end{aligned}
$$

with the two different texture averages of the (same) orientation tensor $T$, Eq. (25). It is

$$
\begin{aligned}
& \bar{T}_{i j k l m n o p}^{\text {Voigt }}=\oint_{g} T_{i j k l m n o p}(g) \cdot f(g) \mathrm{d} g \quad(\text { Voigt }), \\
& \bar{T}_{i j k l m n o p}^{\text {Reuss }}=\int_{\vec{y} \perp(h k l)} T_{i j k l m n o p}(g) \cdot f(g) \mathrm{d} \psi \quad \text { (Reuss). }
\end{aligned}
$$

In the Voigt approximation the texture average is to be taken over all crystal orientations $g$ whereas in the Reuss approximation it is only to be taken over the one-dimensional "path" $\vec{y} \perp(h k l)$ in the orientation space $g$. This is the same path as for the "texture factor", i.e. the normal pole figure, Eq. (4).

Finally, the actual lattice strain pole figure is assumed to be the average of the limiting cases Eqs. $(30 \mathrm{a}, \mathrm{b})$. This is called the Hill approximation:

$$
\tilde{\varepsilon}_{(h k l)}(\vec{y})^{\text {Hill }}=\frac{1}{2}\left[\tilde{\varepsilon}_{(h k l)}(\vec{y})^{\text {Voigt }}+\tilde{\varepsilon}_{(h k l)}(\vec{y})^{\text {Reuss }}\right] \quad \text { (Hill). }
$$

In the Voigt approximation the sample direction $\vec{y}=\{\alpha \beta\}$ enters the expression (30a) only in the second power by the product $y_{i} \cdot y_{j}$. This corresponds to a direction dependence of the strain according to an ellipsoid and this, in turn, expresses itself in the well-known " $\sin ^{2} \psi$ Law" (which in our terminology, is a $\sin ^{2} \alpha$ Law). In the Reuss approximation also the tensor $\bar{T}^{\text {Reuss }}$ depends on the sample direction $\vec{y}$ (as well as on the lattice plane $(h k l)$ ). Hence, the direction dependence in the lattice strain pole figure is of higher order and the " $\sin ^{2} \psi$ Law" is no longer valid. This is illustrated very drastically in Fig. 13 which is adapted from Brakman (1988).

\section{INDEXING UNKNOWN POWDER DIFFRACTION DIAGRAMS}

The reciprocal lattice of a single crystal is defined by the vectors $r_{(h k l)}^{*}$ which must be known according to their absolute values $\left|r_{(h k l)}^{*}\right|$ as well as to their directions $\left\{\Theta_{(h k l)} \gamma_{(h k l)}\right\}$. They can be measured, e.g. by a single crystal diffractometer. This way the indices $(h k l)$ are immediately given (at least with respect to the (arbitrarily) chosen crystal coordinate system).

The reciprocal space of a random powder sample is spherically symmetric, Fig. 2(c). Hence, the intensity depends only on $\left|r_{(h k l)}^{*}\right|$. Indexing a (random) powder diffraction diagram is then the task of finding a reciprocal lattice $r_{(h k l)}^{*}$ which gives 


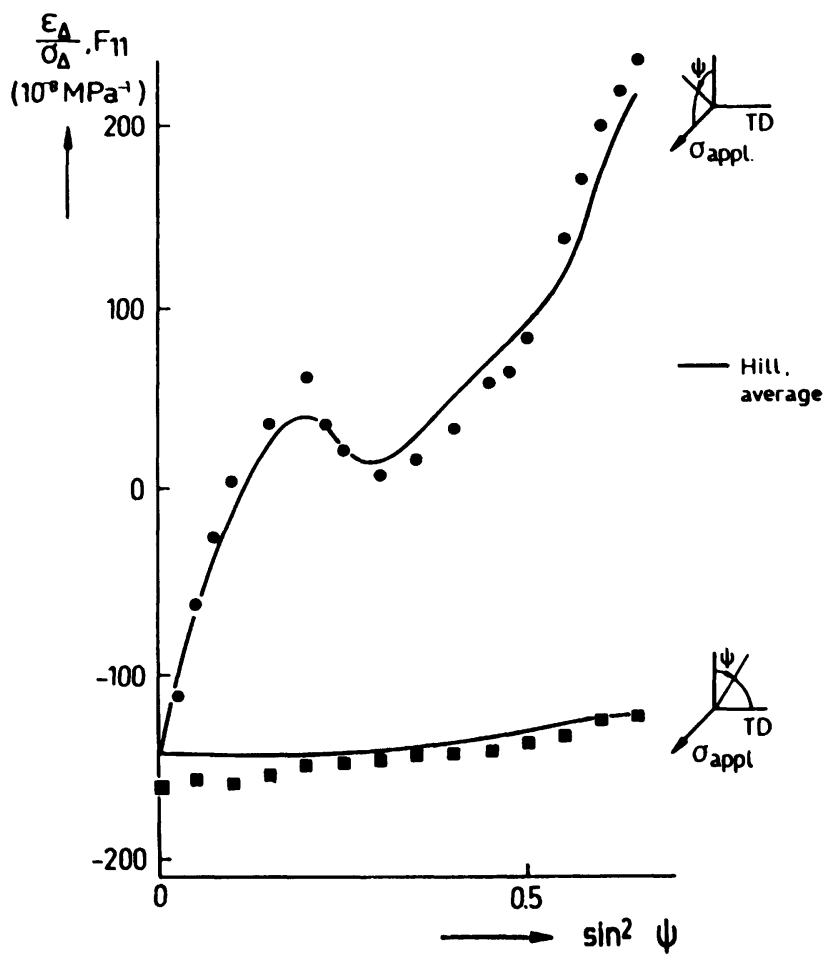

Figure 13 Lattice strain versus $\sin ^{2} \psi$ (corresponding to $\sin ^{2} \alpha$ in the present terminology) in two sample directions compared with the Hill approximation, Eq. (32) (Brakman, 1988).

rise to the absolute values $\left|r_{(h k l)}^{*}\right|$ obtained from the powder diffraction diagram. The solution of this problem must be found in the nine-dimensional space of three reciprocal lattice vectors $\vec{a}^{*} \vec{b}^{*} \vec{c}^{*}$.

The reciprocal space of a textured polycrystalline sample is not spherically symmetric, Fig. 2(b). Rather, the intensity distribution $P_{(h k l)}(\alpha \beta)$ can, in principle, be obtained. Hence, much more experimental data are available to solve the indexing problem. The solution should be the easier the sharper the texture of the sample (in fact, the upper limit of a "sharp texture" is the single crystal). The functions $P_{(h k l)}(\alpha \beta)$ are two-dimensional projections of the three-dimensional texture function $f(g)$ taken along "paths" specified by $(h k l)$, Eq. (4). In fact, the function $f(g)$ depends on three angular variables $\varphi_{1}, \phi, \varphi_{2}$ whereas the projection $P$ depends on four, i.e. $\Theta_{(h k l)} \gamma_{(h k l)} \alpha \beta$ as indicated in Eq. (4). This means that the functions $P$ (of these four variables) cannot be chosen completely deliberately (even not in the case that $f(g)$ is unknown and hence is deliberate). This internal condition between different functions $P_{(h k l)}(\alpha \beta)$ can be used as a criterion for correct (or incorrect) 


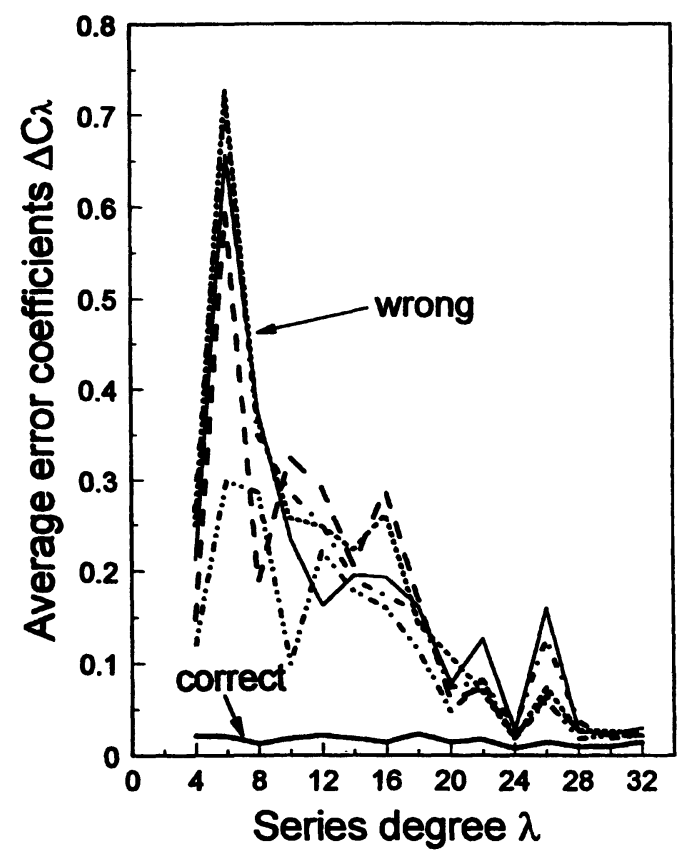

Figure 14 The error quantities $\Delta C_{\lambda}$ for cold rolled copper for correct and incorrect indexing (Bunge and Park, 1996).

indexing. We define a misfit parameter

$$
\sum_{(h k l)} \oint_{\alpha \beta}\left[P_{(h k l)}^{\operatorname{Exp}}(\alpha \beta)-P_{(h k l)}^{\operatorname{Mod}}(\alpha \beta)\right]^{2} \sin \alpha \mathrm{d} \alpha \mathrm{d} \beta=\overline{\Delta P}
$$

which depends on experimental errors of the $P^{\operatorname{Exp}}$ as well as on the correct or incorrect choice of the set of $(h k l)$ in $P^{\text {mod }}$ according to Eq. (4). If the indexing was correct then texture analysis as described above would give the correct texture function $f(g)$ (or a good approximation to it). In this case the misfit parameter would be small. If, on the other hand, indexing was incorrect then the procedure of texture analysis is carried out with wrong coefficients $K_{\lambda}^{\mu}\left(\Theta_{(h k l)} \gamma_{(h k l)}\right)$ in Eq. (8) and it will thus result in a wrong (meaningless) function $f(g)$. Hence, in this case the misfit parameter $\overline{\Delta P}$ will be high.

If the texture is represented in terms of the series expansion (7) then also the misfit parameter $\overline{\Delta P}$ can be expressed in terms of a series expansion (see e.g. Bunge and Park, 1996). In Fig. 14 an example of correct and wrong indexing is shown. The correct indexing is very distinctly distinguished from all wrong indexings. 


\section{References}

Barral, M., Lebrun, J. L., Sprauel, J. M. and Maeder, G. (1987), X-ray stress analysis on textured materials. Calculations of X-ray compliances with the ODF. In: Theoretical Methods of Texture Analysis, Ed. H. J. Bunge, DGM Informationsgesellschaft, Oberursel, 355-364.

Bish, D. L. and Post, J. E. (Eds.) (1989), Modern powder diffraction, Reviews in Mineralogy 20, The Minerlogical Society of America.

Bonarski, J. T., Wcislak, L. and Bunge, H. J. (1994), Investigation of inhomogeneous textures of coatings and near-surface layers, Proc. ICOTOM-10, Materials Science Forum, 157-162, 111-118.

Bonarski, J., Wrobel, M. and Pawlik, K. (1991), Improvement of quantitative phase analysis of textured materials, Scripta Met. et. Mat., 25, 1401-1404.

Brakman, C. M. (1987), Application of the ODF to residual stress analysis problems of textured cubic mateials. Diffraction strain pole figures. In: Theoretical Methods of Texture Analysis, Ed. H. J. Bunge, DGM Informationsgesellschaft, Oberursel, 377-390.

Brakman, C. M. (1988), On the integration of diffraction line shift measurements on textured cubic materials, Thesis Univ. Delft.

Brokmeier, H. G. (1991), Quantitative phase analysis of textured materials, Proc. EPDIC-1, Materials Science Forum, 79-82, 67-72.

Bunge, H. J. (1977), Determination of the orientation distribution function from isolated axis density values, Texture of Crystalline Solids, 2, 169-174.

Bunge, H. J. (1982), Texture Analysis in Materials Science, Gordon and Breach Publ. London, 2nd ed. Cuvillier Verlag Göttingen (1993).

Bunge, H. J. (1988), Texture and directional properties of materials. In: Directional Properties of Materials, ed. H. J. Bunge, DGM Informationsgesellschaft, Oberursel, 1-63.

Bunge, H. J. (1989), Advantages of neutron diffraction in texture analysis, Textures and Microstructures, 10, 265-308.

Bunge, H. J. (1990), Partial texture analysis, Textures and Microstructures, 12, 47-63.

Bunge, H. J. (1991), Preferred orientation in powder diffraction, Proc. EPDIC-1, Materials Science Forum, 79-82, 169-178.

Bunge, H. J. (1994), Influence of texture on powder diffraction, Southern Africa Powder Diffraction Workshop PDSA-94, South African Crystallographic Society.

Bunge, H. J. (1997), Texture analysis. In: Microstructure Analysis from Diffraction, Eds. R. L. Snyder, J. Fiala and H. J. Bunge, Oxford University Press (in print).

Bunge, H. J., Dahms, M. and Brokmeier, H. G. (1989), The determination of integrated intensities from polycrystalline samples with preferred orientation, Crystallography Review, 2, 67-88.

Bunge, H. J. and Park, N. J. (1996), A criterion for correct indexing of powder diffraction diagrams based on preferred orientation (texture), Proceeding of EPDIC-IV Materials Science Forum, 228-231, 25-28.

Bunge, H. J. and Wang, F. (1987), Computational problems in low resolution texture analysis. In: Theoretical Methods of Texture Analysis, Ed. H. J. Bunge, DGM Informationsgesellschaft, Oberursel, $163-172$.

Dahlem-Klein, E., Klein, H. and Park, N. J. (1993), Program system ODF analysis. Cuvillier Verlag, Götingen.

Dahms, M. and Bunge, H. J. (1989), The iterative series expansion method for quantitative texture analysis, J. Appl. Cryst., 22, 439-447.

Dollase, W. A. (1986), Correction of intensities for preferred orientation in powder diffractometry: Application of the March model, J. Appl. Cryst., 19, 267-272.

Fiala, J. (1993), X-ray diffraction depth profiling. In: Hasek (1993) 213-226.

Gao, B., Bunge, H. J., Fantner, R. A. and Van der Veen, R. A. (1992), Texture development in powder mixtures, Advances in $X$-ray Analysis, 35, 321-327.

Gao, B., Bunge, H. J. and Fantner, E. (1993), Estimation of the texture correction in X-ray phase analysis, Proc. EPDIC-2, Materials Science Forum, 133-136, 63-68.

Hasek, J. (1993), Diffraction Methods in Materials Science, Nova Science Publishers, New York.

Hedel, R., Bunge, H. J. and Reck, G. (1997), Model calculations of the accuracy of structure factor determination from textured powder samples, Textures and Microstructures (this volume).

Hoffmann, J., Maurer, G., Neff, H., Scholtes, B. and Macherauch, E. (1986), A PSD-diffractometer for the determination of texture and lattice deformation pole figures. In: Experimental Techniques of Texture Analysis, Ed. H. J. Bunge, DGM Informationsgesellschaft, Oberursel, 409-418.

Järvinen, M., Merisalo, M., Personen, A. and Inkinen, O. (1970), Correction of integrated X-ray intensities for preferred orientation in cubic powders, J. Appl. Cryst., 3, 313-318.

Jenkins, R. and Snyder, R. L. (1996), X-ray Powder Diffractometry, Wiley-Interscience Publication, New York. 
Kiewel, H., Bunge, H. J. and Fritsche, L. (1995), Elastic properties of statistically equivalent materials with varying individual grain orientations, Textures and Microstructures, 25, 17-24.

Klug, H. P. and Alexander, L. E. (1962), X-ray Diffraction Procedures, Wiley-Interscience Publication, New York.

March, A. (1932), Mathematische Theorie der Regelung nach der Korngestalt bei affiner Drehung, Z. Kristallographie, 81, 285-297.

Matthies, S. (1996), Moment pole figures in residual stress analysis, Textures and Microstructures, 25, 229-236.

Reefman, D. (1997), Calculation of the effective absorption coefficient in multi-phase powders, Texture and Microstructures (this volume).

Valvoda, V. (1986), Texture correction in phase analysis and thin film studies, Powder Diffraction, 1, 2832.

Valvoda, V. (1987), Correction of integrated X-ray intensities for preferred orientation in quantitative phase analysis, J. Appl. Cryst., 20, 453-456.

Wcislak, L. and Bunge, H. J. (1996), Program System: Texture Analysis with a Position Sensitive Detector, Cuvillier Verlag Göttingen. 